\title{
Perioperative Management of a Patient with Hypokalemic Periodic Paralysis: A Case Report
}

\author{
Sung Il Bae a,*, Yeran Hwang a , Jongwon Kim a , Seongyeong Tak a Ju-Tae Sohn a,b,c \\ ${ }^{a}$ Department of Anesthesiology and Pain Medicine, Gyeongsang National University Hospital, Jinju, Korea \\ ${ }^{b}$ Department of Anesthesiology and Pain Medicine, Gyeongsang National University College of Medicine, Jinju, Korea \\ ${ }^{c}$ Institute of Health Sciences, Gyeongsang National University, Jinju, Korea
}

\section{Article history:}

Received: January 30, 2020

Revised: April 3, 2020

Accepted: April 6, 2020

\section{${ }^{*}$ Corresponding Author:}

\section{Sung II Bae}

Department of Anesthesiology and Pain Medicine, Gyeongsang National University Hospital, 79 Gangnam-ro, Jinju, Korea

Email: snugsoul@naver.com

\section{ORCID}

Sung II Bae

https://orcid.org/0000-0002-2991-2746

Yeran Hwang

https://orcid.org/0000-0001-5909-664X

Jongwon Kim

https://orcid.org/0000-0003-4137-7333

Seongyeong Tak

https://orcid.org/0000-0001-6609-1986

Ju-Tae Sohn

https://orcid.org/0000-0003-0102-5800

\begin{abstract}
Potassium imbalances can be life-threatening and must be identified and corrected prior to surgery. Patients with hypokalemic periodic paralysis (hypoKPP) experience recurrent muscle weakness or paralysis due to hypokalemia. We present the management of a rare case of hypoKPP during surgery and discuss the general complications and perioperative management of the condition. A 70-year-old man with hypoKPP visited the emergency room with abdominal pain requiring a cholecystectomy. He had not experienced hypoKPP since 1993, 1 year after diagnosis. Preoperative examinations were normal, with a serum potassium level of $4.5 \mathrm{mEq} / \mathrm{L}$. Surgery and recovery were uneventful, with potassium levels $\geq 3.3 \mathrm{mEq} / \mathrm{L}$. The post-surgery serum potassium level was $4.3 \mathrm{mEq} / \mathrm{L}$. The patient had no signs of hypokalemia until 1-week post-surgery. Thorough preoperative preparation, careful assessment of serum potassium levels, avoidance of triggering factors, and appropriate postoperative pain relief can help prevent a hypokalemic attack in patients with hypoKPP.
\end{abstract}

Keywords: hypokalemic periodic paralysis, perioperative care, potassium.

\section{Introduction}

Hypokalemia is an electrolyte imbalance characterized by low serum potassium concentrations (normal range: 3.5-5.0 mEq/L). Symptoms of hypokalemia usually do not present until serum potassium levels are below $3.0 \mathrm{mEq} / \mathrm{L}$. Hypokalemia affects kidney function and is associated with metabolic acidosis and rhabdomyolysis. If the nervous system is affected, the patient may experience weakness, leg cramps, and paralysis. Intestinal paralysis or respiratory difficulties are signs of severe hypokalemia. The effects of hypokalemia on the cardiovascular system present as abnormal electrocardiogram (ECG) findings, cardiac arrhythmia, and heart failure [1-3].

Severe hypokalemia leads to unstable arterial blood pressure caused by arrhythmia, reduced heart rate, respiratory failure, and autonomic dysfunction; hence, careful monitoring, and thorough management of the patient's disease, and nutritional status are essential.

Hypokalemic periodic paralysis (hypoKPP) is a rare, autosomal dominant channelopathy characterized by recurrent attacks of muscle weakness or paralysis owing to associated hypokalemia [4,5]. We report a case of hypoKPP in a patient who underwent laparoscopic cholecystectomy and discuss the perioperative management.

The study was approved by the Institutional Review Board (no.: 2019-09-003). The patient provided written consent for the publication of the data. 


\section{Case Report}

A 70-year-old male patient (of height $158.9 \mathrm{~cm}$, and weight $57.9 \mathrm{~kg}$ ) with a previous diagnosis of hypoKPP visited the emergency room with abdominal pain which began the day before. In 1992, he was diagnosed with hypoKPP following weakness of the limbs and recovery from palpitations after 5 minutes of symptoms. He reported that his physician instructed him to consume potassium-rich food and he was not prescribed any medications. Symptoms recurred for 1 year; thereafter, no hypokalemia-associated adverse event was noted, including weakness or palpitations. He had been diagnosed with hypertension 20 years ago and orthostatic hypotension in February 2017. There was no history of thyroid disease, and no specific findings in the thyroid function tests in 2007,2009 , and 2015. The patient was diagnosed with calculus of the gallbladder with acute cholecystitis. Ceftriaxone ( $2 \mathrm{~g}$ ) and fentanyl $(100 \mu \mathrm{g})$ were administered intravenously. A cholecystectomy was performed the following day.

On preoperative physical examination, muscle strength in all extremities was normal. Breathing was clear bilaterally on auscultation. Airway assessment was normal: Mallampati Class 1 with normal neck and jaw movement. The initial heart rate was 80 beats/minute, and the blood pressure was 150/76 mmHg. Laboratory results showed hemoglobin levels of $14.2 \mathrm{~g} /$ $\mathrm{dL}$, serum potassium levels of $4.5 \mathrm{mEq} / \mathrm{L}$, normal liver function, and normal coagulation parameters. The chest radiograph and ECG showed no abnormalities. Upon arrival to the operation room, monitors were set up including the ECG, non-invasive blood pressure, and pulse oximetry. The anesthesiologist performed cannulation of the radial artery to assess the potassium level and monitor the blood pressure, prepared potassium for correction of hypokalemia, and excluded glucosecontaining fluid. The anesthesiologist attached a bispectral index to maintain the appropriate depth of general anesthesia. Total intravenous anesthesia was achieved using a targetcontrolled infusion pump with propofol and remifentanil after intravenous injection of lidocaine ( $30 \mathrm{mg}$ ). The target plasma concentration of propofol was maintained at $4 \mu \mathrm{g} / \mathrm{mL}$, and the

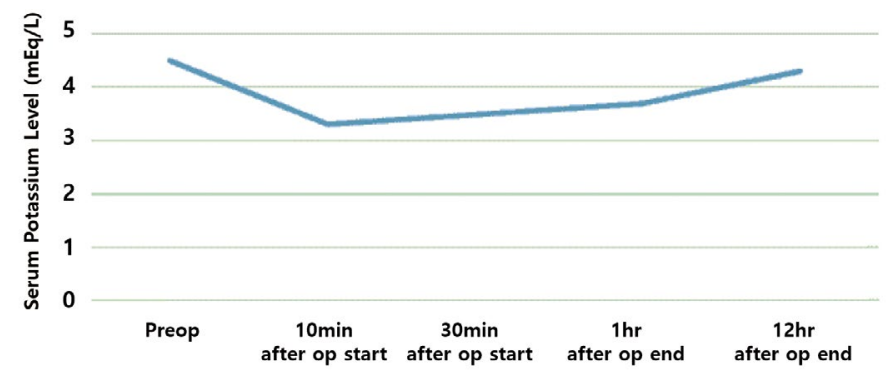

Figure 1. Perioperative potassium levels. target plasma concentration of remifentanil was maintained at $3 \mu \mathrm{g} / \mathrm{mL}$. To avoid hypocapnia, bag-mask ventilation was performed to maintain end-tidal pressure of carbon dioxide at $35-40 \mathrm{mmHg}$. He was intubated with a $7.5 \mathrm{~mm}$ endotracheal tube. An oropharyngeal temperature probe was inserted for temperature monitoring during the operation. To maintain body temperature, warming fluid, Blanketrol (Cincinnati Sub-Zero, Cincinnati, OH), Bair Hugger (Augustine Medical, Courtelary, Switzerland), and heat and moisture exchanger filters were used. The total anesthesia time was 1 hour and 15 minutes; at 15 minutes after the start of the operation, the potassium level was $3.3 \mathrm{mEq} / \mathrm{L}$ on arterial blood gas analysis and was $3.5 \mathrm{mEq} / \mathrm{L}$ after 30 minutes. The hemodynamics remained stable throughout the operation, with a heart rate of $60-80$ beats/minute, a mean blood pressure of $80-95 \mathrm{mmHg}$, and a body temperature of $36.5-36.8^{\circ} \mathrm{C}$. The intraoperative blood loss was less than $50 \mathrm{~mL}$. Ten minutes before the end of the operation, $50 \mathrm{mg}$ of fentanyl was administered to control pain. After the operation, the tidal volume of the patient was $>350 \mathrm{~mL}$. After confirming conscious recovery and limb movement, the patient was extubated. The patient's condition was evaluated, and he was transferred to the recovery room where his pain level was assessed using a numerical rating scale 3 to 5 . There were no specific symptoms of muscle weakness or throbbing; therefore, he was moved to the ward with a warm blanket in accordance with the criteria for leaving the recovery room. After surgery, the serum potassium level was $4.3 \mathrm{mEq} / \mathrm{L}$, and there were no other laboratory result abnormalities. To control the patients pain whilst on the ward, an opioid analgesic (Conofen; Hana pharm, Seoul, Korea) 1 capsule (acetaminophen $250 \mathrm{mg}$, ibuprofen $200 \mathrm{mg}$, codeine phosphate $10 \mathrm{mg}$ ) was administered twice daily, and the pain level was maintained (below an numerical rating scale score 3 ). There were no symptoms similar to hypokalemia until 1 week after the day of operation (Figure 1).

\section{Discussion}

HypoKPP is an autosomal dominant inherited disorder characterized by flaccid paralysis and proximal muscle weakness [4,5]. Hypokalemia mechanisms are considered to be related to inappropriate absorption of potassium by muscle cells, leading to a decreased plasma potassium concentration $[6,7]$. When potassium is removed from the cell and serum potassium levels are normalized, the symptoms resolve spontaneously. A patient with hypoKPP is at an increased risk of perioperative hypokalemic paralysis. Severe preoperative paralytic attacks may require intubation [4]. Factors triggering paralytic attacks include high carbohydrate meals, meals with high sodium content, alcohol, infection, menstruation, 
pregnancy, glucose infusion, insulin, stressful conditions (e.g., surgery, pain, hypothermia, fear, noise), excitement, metabolic or respiratory alkalosis, anesthesia, steroids, and prolonged immobility [1,4-7].

It is safe to perform surgery when the patient's serum potassium level is normal (3.5-5.0 mEq/L) however, in patients with hypoKPP, the safe serum potassium level before surgery varies from that in normal patients. In case of a low normal serum potassium level, the probability of a hypokalemic attack increases, and the symptoms may be more severe during a hypokalemic attack. Therefore, the surgeon must perform the surgery after replacement of the serum potassium levels to achieve a high normal level. The general potassium replacement protocol recommends correction of the patient's serum potassium level to $5.0 \mathrm{mEq} / \mathrm{L}$ before and after surgery. Surgeons and anesthesiologists should recognize these triggering factors and avoid them when patients are scheduled for surgery. To prevent hypokalemic paralytic attacks, dextrose-containing fluid and insulin should be avoided. Epidural block, transverse abdominal plane block, and opioids are necessary for appropriate pain control during surgery. Bispectral index monitoring should be performed to maintain the correct depth of anesthesia. To prevent hypothermia, body temperature should be maintained using a warming fluid, hypo/hyperthermia machines, and heat and moisture exchanger filters. The end-tidal carbon dioxide monitoring is needed to prevent respiratory alkalosis. Arterial cannulation is essential to monitor hemodynamic instability induced by hypokalemia and to measure serum potassium levels. The relationship between malignant hyperthermia and hypoKPP has never been definitively demonstrated however, surgeons and anesthesiologists must be aware of the possibility and be prepared to manage malignant hyperthermia [8]. In cases where patient medical history is not known, thyroid function tests should be performed to rule out hyperthyroidism, a common condition among Asian men that may lead to thyrotoxic periodic paralysis.

In this case, the patient's hypokalemic attack may have been caused by operative stress, pain, or anesthesia among other trigger factors. The patient was observed (without receiving potassium supplements) when the potassium level was 3.3 $\mathrm{mEq} / \mathrm{L}$ in the first arterial blood gas, because the heart rate (63 beats/minutes), blood pressure $(125 / 60 \mathrm{mmHg})$, body temperature $\left(36.9^{\circ} \mathrm{C}\right)$, and $\mathrm{pCO} 2(41)$ were within normal limits. In addition, there was no evidence of a typical ECG pattern of hypokalemia, such as flattened $\mathrm{T}$ waves, prominent waves, ST segment depression, or a fusion of $\mathrm{T}$ and $\mathrm{U}$ waves. The general potassium replacement protocol was not performed, and the postoperative potassium level was 4.3 $\mathrm{mEq} / \mathrm{L}$. Although potassium levels were not replenished, they rose to preoperative levels. Surgical trauma itself can increase potassium levels after surgery however, the minimally invasive surgery in this case was not considered to have a major impact. The main cause for the increase in potassium levels was likely to be the mechanism of this disease, with the potassium that entered the muscle cells being released back into the blood $[1,6,7]$.

We were prepared to prevent an attack of hypokalemia owing to the patient's condition however, hypokalemia occurred during surgery, with the potassium level falling to $3.3 \mathrm{mEq} / \mathrm{L}$. Whilst reviewing this case, several other ways to prevent hypokalemic attacks were determined that had not been considered. If preoperative transverse abdominis plane block and midazolam premedication had been performed, stress and the possibility of a hypokalemic attack could have been reduced $[9,10]$. All of this should be managed from the time of the patient's arrival until the time of discharge from hospital.

In conclusion, in the management of a patient with hypoKPP undergoing laparoscopic cholecystectomy, surgeons and anesthesiologists should be aware that hypokalemia can occur suddenly during surgery. Thorough preoperative preparation, careful assessment of serum potassium levels, avoidance of triggering factors, and appropriate postoperative pain relief can help prevent a hypokalemic attack in patients with hypoKPP.

\section{Conflicts of Interest}

The authors have no conflicts of interest to declare.

\section{References}

[1] Kardalas E, Paschou SA, Anagnostis P, Muscogiuri G, Siasos G, Vryonidou A. Hypokalemia: A clinical update. Endocr Connect 2018;7(4):R135-46.

[2] Zacchia M, Abategiovanni ML, Stratigis S, Capasso G. Potassium: From physiology to clinical implications. Kidney Dis (Basel) 2016;2(2):72-9.

[3] Marti G, Schwarz C, Leichtle AB, Fiedler GM, Arampatzis S, Exadaktylos $\mathrm{AK}$, et al. Etiology and symptoms of severe hypokalemia in emergency department patients. Eur J Emerg Med 2014;21(1):46-51.

[4] Viscomi CM, Ptacek LJ, Dudley D. Anesthetic management of familial hypokalemic periodic paralysis during Parturition. Anesth Analg 1999;88(5):1081-2.

[5] Bandschapp O, Iaizzo PA. Pathophysiologic and anesthetic considerations for patients with myotonia congenita or periodic paralyses. Paediatr Anaesth 2013;23(9):824-33.

[6] Lema G, Urzua J, Moran S, Canessa R. Successful anesthetic management of a patient with hypokalemic familial periodic paralysis undergoing cardiac surgery. Anesthesiology 1991;74(2):373-5.

[7] Chitra S, Korula G. Anaesthetic management of a patient with hypokalemic periodic paralysis- a case report. Indian J Anaesth 2009;53(2):226-9.

[8] Lambert C, Blanloeil Y, Horber RK, Berard L, Reyford H, Pinaud M. Malignant hyperthermia in a patient with hypokalemic periodic paralysis. Anesth Analg 1994;79(5):1012-4.

[9] Mitra S, Khandelwal P, Roberts K, Kumar S, Vadivelu N. Pain relief in laparoscopic cholecystectomy-A review of the current options. Pain Pract 2012;12(6):485-96.

[10] Bauer KP, Dom PM, Ramirez AM, O'Flaherty JE. Preoperative intravenous midazolam: Benefits beyond anxiolysis. J Clin Anesth 2004;16(3):177-83. 